\title{
INTELLIGENT PERFORMANCE ANALYSIS WITH A NATURAL LANGUAGE INTERFACE
}

\author{
Esko K. JUUSO
}

University of Oulu

\begin{abstract}
:
Performance improvement is taken as the primary goal in the asset management. Advanced data analysis is needed to efficiently integrate condition monitoring data into the operation and maintenance. Intelligent stress and condition indices have been developed for control and condition monitoring by combining generalized norms with efficient nonlinear scaling. These nonlinear scaling methodologies can also be used to handle performance measures used for management since management oriented indicators can be presented in the same scale as intelligent condition and stress indices. Performance indicators are responses of the process, machine or system to the stress contributions analyzed from process and condition monitoring data. Scaled values are directly used in intelligent temporal analysis to calculate fluctuations and trends. All these methodologies can be used in prognostics and fatigue prediction. The meanings of the variables are beneficial in extracting expert knowledge and representing information in natural language. The idea of dividing the problems into the variable specific meanings and the directions of interactions provides various improvements for performance monitoring and decision making. The integrated temporal analysis and uncertainty processing facilitates the efficient use of domain expertise. Measurements can be monitored with generalized statistical process control (GSPC) based on the same scaling functions.
\end{abstract}

Key words: data analysis, nonlinear scaling, trend analysis, fuzzy systems, natural language

\section{INTRODUCTION}

Advanced data analysis is used to integrate process and condition monitoring measurements. Dimensionless indices, which are obtained by comparing each feature value with the corresponding value in normal operation, provide useful information on different faults, and even more sensitive solutions can be obtained by selecting suitable features [1]. Generalized moments and norms include many wellknown statistical features as special cases and provide compact new features capable of detecting faulty situations. A combination of real order derivatives and generalized norms [2] can be used in various applications [3]. Intelligent indices are developed from these features by the databased nonlinear scaling introduced in [4].

There are many ways to measure, monitor and analyse maintenance and operation performance [5], e.g. harmonized indicators $[6,7]$, key performance indicators (KPI) $[8$, 9] and overall equipment effectiveness (OEE) $[10,11]$ provide useful numeric values. Willmott presents several examples of OEE improvements with examples of financial benefits [12].

Trend analysis systems have three components: a language to represent the trends, a technique to identify the trends, and mapping from trends to operational conditions [13]. The fundamental elements are modelled as triangles to describe local temporal patterns. The elements are defined by the signs of the first and second derivative, respectively. They are also known as triangular episodic representations [14].

Changing operating conditions need to be taken into account in prognostics since new phenomena activate grad- ually with time. In the condition-based maintenance (CBM), the most obvious and widely used form of prognostics is to predict how much time is left before a failure occurs. The time left before a failure is usually called remaining useful life (RUL) [15]. The wear conditions collected up to the current inspection are used in [16] to define the time for the next inspection. Wang compared Weibull and Gamma distributions in parameter estimation [17]: the distribution of residual time starts from the normal distribution and moves through skew distributions to a very narrow distribution when an actual failure progresses.

Fatigue is progressive, localised structural damage caused by repeated loading and unloading. The history of the analysis already began in 1837, when Wilhelm Albert published the first fatigue test results [18]. Wöhler concluded that cyclic stress range is more important than peak stress and introduced the concept of the endurance limit. The effects of each stress level are taken into account in the calculations of cumulative damage from individual contributions $[19,20]$.

Fuzzy set theory first presented by Zadeh form a conceptual framework for linguistically represented knowledge [21]. The extension principle is the basic generalisation of the arithmetic operations if the inductive mapping is a monotonously increasing function. Several fuzzy modelling approaches can be combined: fuzzy arithmetics are suitable both for processing the fuzzy inputs and outputs of the rule -based fuzzy set system; fuzzy inequalities produce new facts; fuzzy relations can be represented as the sets of alternative rules, where each rule has a degree of membership [22]. 
This paper focuses on the methodologies of developing intelligent performance measures based on the nonlinear scaling of measurements and domain expertise. The solutions aimed for performance monitoring and decision making is enhanced with temporal analysis, uncertainty processing and a natural language interface.

\section{DATA ANALYSIS}

Detecting operating conditions and faults can be based on data analysis of various types of measurements (Fig. 1). The feedback information comes from performance indices. Nonlinear scaling brings all these to the same scale as numeric values and linguistic meanings.

\section{Features}

Normalisation or scaling of the data is needed since measurements with considerably different magnitudes cause problems in modelling. The nonlinear scaling extends modelling to various statistical distributions and allows recursive tuning.

Arithmetic mean and standards deviation, which are the key statistical features in industrial practice, are special cases of generalized norms

$$
\left\|\tau M_{j}^{p}\right\|_{p}=\left({ }^{\tau} M_{j}^{p}\right)^{1 / p}=\left[\left(\frac{1}{N} \sum_{i=1}^{N}\left(x_{j}\right)_{i}^{p}\right)\right]^{1 / p},
$$

where the order of the norm $p \in \mathfrak{R}$ is non-zero. The analysis is based on consecutive equally sized samples. Duration of each sample is called sample time, denoted $\tau$, and $N$ is the number values in the sample. For waveform signals, the number of signal values $N=\tau N_{S}$, where $N_{S}$ is the number of signal values which are taken in a second. The norm (1) has the same dimensions as the signals $N_{S}$, where $\alpha$ is the order of derivation, e.g. $\alpha=2$ for widely used acceleration signals. The analysis can also use derivated signals. The generalized norms were introduced for condition monitoring
$[2,3]$. The norm values increase monotonously with increasing order if all the signals are not equal.

The computation of the norms can be divided into the computation of equal sized sub-blocks, i.e. the norm for several samples can be obtained as the norm for the norms of individual samples. The same result is obtained using the norms of the sub-blocks:

$$
\left\|K_{S} \tau M_{\alpha}^{p}\right\|_{p}=\left\{\frac{1}{K_{S}} \sum_{i=1}^{K_{S}}\left[\left({ }^{\tau} M_{\alpha}^{p}\right)_{i}^{1 / p}\right]^{p}\right\}^{1 / p}=\left[\frac{1}{K_{S}} \sum_{i=1}^{K_{S}}\left({ }^{\tau} M_{\alpha}^{p}\right)_{i}\right]^{1 / p},
$$

where $K_{s}$ is the number of samples. Each sample has $\mathrm{N}$ variable values. As the aggregation can be continued to longer and longer time periods, this generalizes the practice used automation systems for the arithmetic means.

High order derivatives of the acceleration signal improve fault detection $[2,3]$. Stress analysis can be done without derivation, but the sensitivity is improved when higher orders $\alpha$ are used. Spectral norms also answer the question of which frequency range the changes are in since they combine the time domain analysis with the frequency domain analysis [24].

\section{Performance indicators}

Harmonized indicators are used for monitoring maintenance actions on a management level, where the indicators are based on cost, time, man-hours, inventory value, work orders and cover of the criticality analysis $[6,7]$.

Key performance indicators (KPIs) are quantifiable measures which reflect the critical success factors and the goals of the organization. KPIs differ depending on the organization and can focus on different parts and levels of the process. Accurately defined and measured KPIs provide feedback information for decision making. The maintenance function covers various aspects, including quality assurance, financial, reliability, planning, execution, strategic, data completeness, logistics and competency [9]. The perfor-

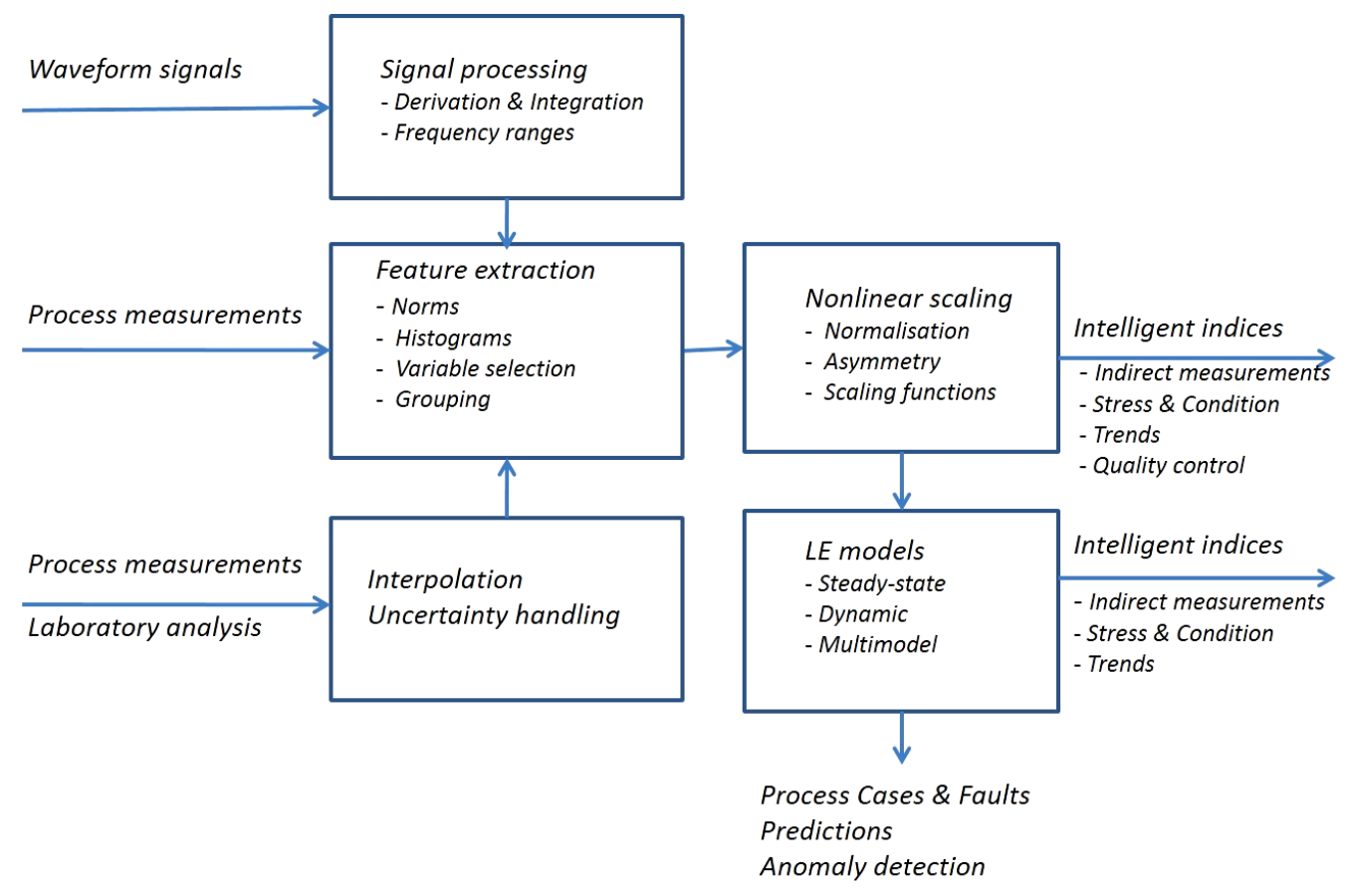

Fig. 1 Detecting operating conditions and faults

Source: [23]. 
mance metrics can be assessed with the SMART criteria: specific, measurable, attainable, realistic and timely [8].

Overall equipment effectiveness (OEE) is a set of broadly accepted non-financial metrics which reflects the manufacturing success by availability (uptime), performance rate and quality rate $[10,11]$.

\section{Nonlinear scaling}

The z-score based linear scaling solutions are extended to asymmetric nonlinear scaling functions defined by two second order polynomials (Fig. 2).
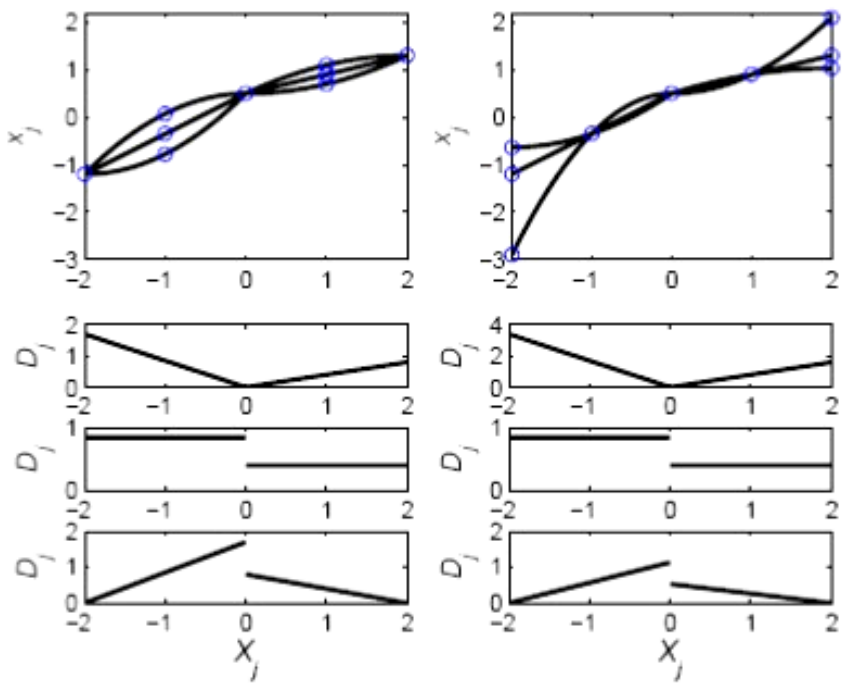

Fig. 2 Feasible shapes of membership definitions $f_{j}$ and corresponding derivatives $D_{j}$ : coefficients adjusted with the core (left) and support (right). Derivatives are presented in three groups: (1) decreasing and increasing, (2) asymmetric linear, and (3) increasing and decreasing Source: [27].

The parameters of the polynomials are defined with five parameters corresponding the operating point $c_{j}$ and four corner points of the feasible range [25]. The feasible range is defined as a trapezoidal membership function defined by support and core areas, see [26]. The scaling functions are monotonously increasing throughout the feasible range, see $[22,27]$. This is satisfied if the coefficients,

$$
\begin{aligned}
& \alpha_{j}=\frac{\left(c_{l}\right)_{j}-\min \left(x_{j}\right)}{c_{j}-\left(c_{l}\right)_{j}}=\frac{\left(c_{l}\right)_{j}-\min \left(x_{j}\right)}{\Delta c_{j}^{-}} \\
& \alpha_{j}^{+}=\frac{\max \left(x_{j}\right)-\left(c_{h}\right)_{j}}{\left(c_{h}\right)_{j}-c_{j}}=\frac{\max \left(x_{j}\right)-\left(c_{h}\right)_{j}}{\Delta c_{j}^{+}},
\end{aligned}
$$

are restricted to the range $\left[\frac{1}{3}, 3\right]$.

The scaled values are obtained by means of the inverse function $f^{-1}$ :

$$
X_{j}=\left[\begin{array}{cl}
2 \text { with } & x_{j} \geq \max \left(x_{j}\right) \\
\frac{-b_{j}^{+}+\sqrt{b_{j}^{+2}-4 a_{j}^{+}\left(c_{j}-x_{j}\right)}}{2 a_{j}^{+}}-2 & \text { with } \quad c_{j} \leq x_{j} \leq \max \left(x_{j}\right) \\
\frac{-b_{j}^{-}+\sqrt{b_{j}^{-2}-4 a_{j}^{-}\left(c_{j}-x_{j}\right)}}{2 a_{j}-2}-2 & \text { with } \quad \min \left(x_{j}\right) \leq x_{j} \leq c_{j} \\
-2 \text { with } & x_{j} \leq \min \left(x_{j}\right)
\end{array}\right.
$$

Where $a_{j}, b_{j}, a_{j}^{+}$and $b_{j}^{+}$are coefficients of the corresponding polynomials represented by

$$
\begin{aligned}
& a_{\bar{j}}=\frac{1}{2}\left(1-\alpha_{\bar{j}}\right) \Delta c_{j}, \\
& b_{\bar{j}}=\frac{1}{2}(3-\alpha \bar{j}) \Delta c_{j}, \\
& a_{j}^{+}=\frac{1}{2}\left(\alpha_{j}^{+}-1\right) \Delta c_{j}^{+}, \\
& b_{j}^{+}=\frac{1}{2}\left(3-\alpha_{j}^{+}\right) \Delta c_{j}^{+},
\end{aligned}
$$

Data-based tuning by using generalized norms and skewness was introduced in [4]. The constraints are taken into account by moving the corner points or the upper and lower limits if needed. The systems can be tuned with genetic algorithms [27].

\section{Intelligent indices}

Intelligent indices are obtained from measurements and features by the nonlinear scaling approach. The indices obtained from short samples are aimed for use in the same way as indirect measurements, e.g. to indicate stress or condition (Fig. 1). Several indices can be combined in linguistic equation (LE) modelling since the indices are dimensionless. Grouping is important for large scale systems [28].

The cavitation index is an example of a stress index [4]: the approach provides four levels whose values ranges

\begin{tabular}{|c|c|c|c|}
\hline \multirow[b]{2}{*}{ Level } & \multicolumn{3}{|c|}{ Severity classification } \\
\hline & $\begin{array}{l}\text { Cavitation } \\
\text { index }\end{array}$ & Cavitation level & Severity \\
\hline 1 & $I_{C}^{(4)}<-1$ & Cavitation-free & Good \\
\hline 2 & $-1 \leq I_{C}^{(4)}<0$ & $\begin{array}{c}\text { Short periods } \\
\text { of weak cavitation }\end{array}$ & Usable \\
\hline 3 & $0 \leq I_{C}^{(4)}<1$ & $\begin{array}{l}\text { Short periods } \\
\text { of cavitation }\end{array}$ & Still acceptable \\
\hline 4 & $I_{C}^{(4)} \geq 1$ & Cavitation & Not acceptable \\
\hline
\end{tabular}
shown in Table 1 are consistent with the limits of the vibration severity ranges defined in $[29,30]$. Strong cavitation can be avoided with better an allocation of the energy production [31].

Table 1

Severity cavitation

Source: [4].

In a hot rolling mill, the stress indices were developed by using torque measurements: the feature is difference between the effective and average values, i.e.

$$
x_{T}=\left[\left(\frac{1}{N} \sum_{i=1}^{N}\left(x_{j}\right)_{i}^{2}\right)\right]^{1 / 2}-\frac{1}{N} \sum_{i=1}^{N}\left(x_{j}\right)_{i},
$$

where $x_{j}$ is the fillet split. The time interval can be different for the passes. Since the orders of the norm are here 1 and 2 , also negative values of $x_{j}$ can be used [32].

Stress indices for the front axle of a load haul dumper (LHD) have been developed from acceleration signals by

using feature $\max \left(\left\|{ }^{5} M_{2}^{4}\right\|\right)$. The analysis provides good indications of different stress contributions in these machines, which operate in harsh conditions where failures may be difficult to repair [33]. The cumulative stress method has recently been used in the monitoring of a rod mill [34, 35]. 
Intelligent indices based on two generalized norms are highly sensitive to faulty situations in the supporting rolls of a lime kiln. Surface damage and misalignment are clearly detected. The data set covers surface problems, good conditions after grinding, misalignment, stronger misalignment, very good conditions after repair work, and very good conditions one year later [4]. Sensitivity is also improved for weak friction and small fluctuations. This is useful in detecting lubrication problems. All the supporting rolls can be analyzed using the same approach throughout the data set. The results are consistent with the vibration severity criteria: good, usable, still acceptable, and not acceptable.

The condition indices of the LHD machine need to be obtained repeatedly in similar steady operating conditions [33]. Extensions to real and complex order derivatives are discussed in [36].

\section{TEMPORAL ANALYSIS}

Fluctuations, trends and models are used in temporal analysis for all types of measurements, features and indices. Recursive updates of the parameters are needed in prognostics.

\section{Fluctuations}

The fluctuations are evaluated as the difference of the high and the low values as a difference of two moving generalized norms:

$$
\Delta x_{j}^{F}(k)=\left\|K_{s} \tau M_{j}^{p_{H}}\right\|_{p_{H}}-\left\|K_{s} \tau M_{j}^{p_{L}}\right\|_{p_{L}},
$$

where the orders $p_{H} \in \mathfrak{R}$ and $p_{L} \in \mathfrak{R}$ are large positive and negative, respectively. The norms are calculated from the latest $K_{\mathrm{s}}+1$ values, and an average of several latest values of $\Delta x_{j}^{F}(k)$ is used as the feature of fluctuation. The feature, which was originally developed for control [37], is easy to calculate and more robust than using the difference of the actual maximum and minimum.

The fluctuation indices are calculated from features (7) by the nonlinear scaling. Similar calculations can be done for intelligent indices if the variations close to the normal conditions are important.

\section{Trend analysis}

For any variable $\mathrm{x}_{\mathrm{j}}$, a trend index $I_{j}^{T}(k)$ is calculated from the scaled values $X_{j}$ with

$$
I_{j}^{T}(k)=w_{j}\left[\frac{1}{\left(n_{S}\right)_{j}+1} \sum_{i=k-\left(n_{S}\right)_{j}}^{k} X_{j}(k)-\frac{1}{\left(n_{L}\right)_{j}+1} \sum_{i=k-\left(n_{L}\right)_{j}}^{k} X_{j}(k)\right]
$$

which is based on the means obtained for a short and a long time period, defined by delays $\left(n_{S}\right)_{j}$ and $\left(n_{L}\right)_{j}$, respectively. The weight is variable specific. The index value is in the linguistic range $[-2,2]$ representing the strength of both the decrease and increase of the variable $x_{j}[38]$.

Episode alternatives are shown in Fig. 3. An increase is detected if the trend index exceed a threshold $I_{j}^{T}(k)>\varepsilon_{1}^{+}$. Correspondingly, $I_{j}^{T}(k)<\varepsilon_{1}^{-}$for a decrease. The derivative of the index $I_{j}^{T}(k)$ denoted as $\Delta I_{j}^{T}(k)$, extends the analysis to nonlinear episodes. Trends are linear if the derivative is close to zero: $\varepsilon_{2}<\Delta I_{j}^{T}(k)<\varepsilon_{2}^{+}$. The concave upward mono- tonic increase (D) and the concave downward monotonic decrease (B) are dangerous situations, which introduce warnings and alarms. The concave downward monotonic increase (A) and the concave upward monotonic decrease (C) mean that a harmful trend is stopping.

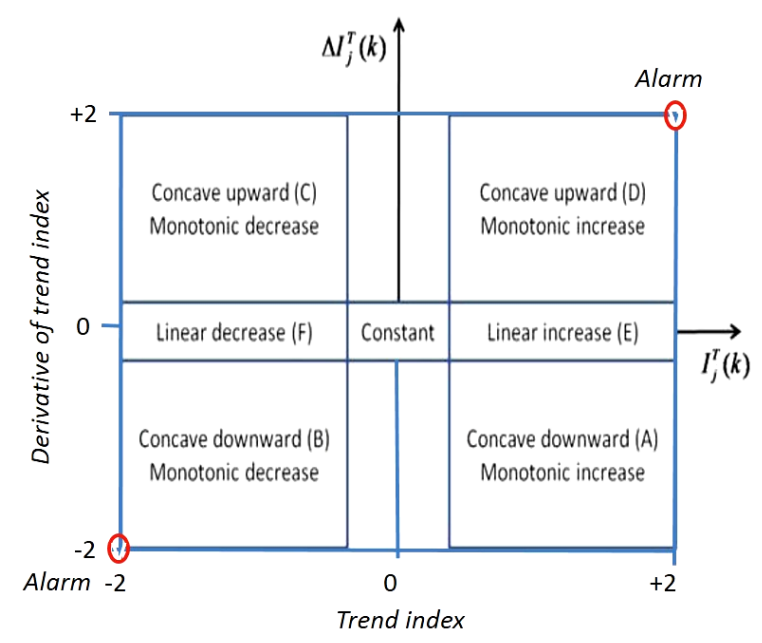

Fig. 3 Intelligent trend analysis Source: [38]. index

Severity of the situation can be evaluated by a deviation

$$
I_{j}^{D}(k)=\frac{1}{3}\left(X_{j}(k)+I_{j}^{T}(k)+\Delta I_{j}^{T}(k)\right),
$$

whose absolute values are the highest when the difference to the set point is very large and is getting still larger with a fast increasing speed.

The trend analysis is tuned to applications by selecting variable specific the time periods $\left(n_{L}\right)_{j}$ and $\left(n_{S}\right)_{j}$. The thresh olds $\varepsilon_{1}^{-}=\varepsilon_{1}^{+}=\varepsilon_{2}=\varepsilon_{2}^{+}=0.5$. Further fine-tuning can be done by adjusting the weight factors $w_{j}^{T 1}$ and $w_{j}^{T 2}$ used for the indices $I_{j}^{T}(k)$ and $\Delta I_{j}^{T}(k)$. The calculations are done with numerical values and the results are represented in natural language [39].

Trend indices can be calculated from the scaled values of measurements and features, intelligent indices and linguistic information. Interpretation in natural language follows the same guidelines.

\section{Prognostics}

In a load haul dumper (LHD), the cumulative stress increases fast during the high stress periods and increase is practically stopped when the stress is low since only stress indices are taken into account in the cumulative stress. [33] Recursive updates of the scaling functions become important in prognostics since the machine or process device is in good condition in the starting point. The rough early estimates are gradually refined if the failure predictions are not yet real. The interaction models are not changed [40].

\section{Risk analysis}

Varying operating conditions have been taken into account in fatigue analysis $[32,41]$ by representing the Wöhler curves with a linguistic equation

$$
I_{S}=\log _{10}\left(N_{C}\right)
$$

where the stress index is the scaled value of stress,

$$
I_{S}=f^{-1}\left(S_{i}\right)=f^{-1}\left(\left\|{ }^{2} M_{\alpha}^{p}\right\|\right)
$$


The scaling of the logarithmic values of the number of cycles, $N_{C}(k)$, is linear. In each sample time, $\tau$, the cycles $N_{C}(k)$ are obtained by (10) and added to the previous contributions by

$$
C(k)=C(k-1)+\frac{\tau}{N_{C}(k)},
$$

where the value range of the sum $\mathrm{C}$ is scaled to provide the fatigue risk in percent (\%).

The high stress contributions dominate in the summation. Correspondingly, the very low stress periods have a negligible effect, which is consistent with the idea of infinite life time. The summation of the contributions also reveals repeated loading and unloading, and the individual contributions provide indications for the severity of the effect. The stress levels can be followed by a generalized statistical process control approach [42]. At the risk level higher than $60 \%$, a single high torque level can have a strong effect on the activation of a failure.

\section{UNCERTAINTY PROCESSING}

Scaling functions developed in data analysis are the basis of the uncertainty processing. All scaled values and fuzzy terms can be interpreted in natural language. The fuzzy interface is also used to introduce additional expert knowledge in the calculations.

\section{Varying operating conditions}

The features and indices are calculated with problemspecific sample times and the variation with time is handled as uncertainty by presenting the indices as timevarying fuzzy numbers. The classification limits can also be considered fuzzy.

The parameters of the scaling functions are specific to operating conditions, some changes can be taken into account switching the parameter sets. The parameters become fuzzy numbers if the time period includes different operating conditions. The results of the fuzzy scaling are fuzzy numbers for crisp values as well. All intelligent indices, including fluctuation, trend and deviation indices, can be presented as fuzzy numbers.

\section{Knowledge-based information}

Domain expertise can include information about levels which can be translated into fuzzy numbers. The labels \{very low, low, normal, high, very high or ffast decrease, decrease, constant, increase, fast increase $\}$ can be represented by number $\{-2,-1,0,1,2\}$. Different shapes of membership definitions result different sets of default membership functions: the locations depend on the core, the support and the centre point. However, the linguistic data can be understood as scaled values, whose membership functions are equally spaced, i.e. $\{-2,-1,0,1,2\}$. The overlap between adjacent linguistic terms expresses a smooth transition from one term to the other [43].

The fuzzy sets can be modified by fuzzy modifiers, which are used as intensifying adverbs (very, extremely) or weakening adverbs (more or less, roughly). The resulting terms, e.g.

$$
\text { extremely } A \subseteq \text { very } A \subseteq A \subseteq \text { more orless } A \subseteq \text { roughly } A \text {, }
$$

correspond to the powers $\{4,2,1,1 / 2,1 / 4\}$ of the membership in the powering modifiers. The vocabulary can also be chosen in a different way, e.g. highly, fairly, quite [43]. Only the sequence of the labels is important. Linguistic variables can be processed with the conjunction (AND), disjunction (OR) and negation (NOT). More examples can be found in [44].

\section{Fuzzy calculus}

Fuzzy calculus is suitable for processing fuzzy inputs and outputs in the rule-based fuzzy set systems, but the rulebased system is not necessarily needed (Fig. 4). The extension principle is the basic generalisation of the arithmetic operations if the inductive mapping is a monotonously increasing function of the input. The interval arithmetic presented by Moore [45] is used together with the extension principle on several membership $\alpha$-cuts of the fuzzy number $x_{j}$ for evaluating fuzzy expressions $[46,47,48]$.

Fuzzy inequalities produce new facts like $A \leq B$ and $A=B$ for fuzzy inputs $A$ and $B$.

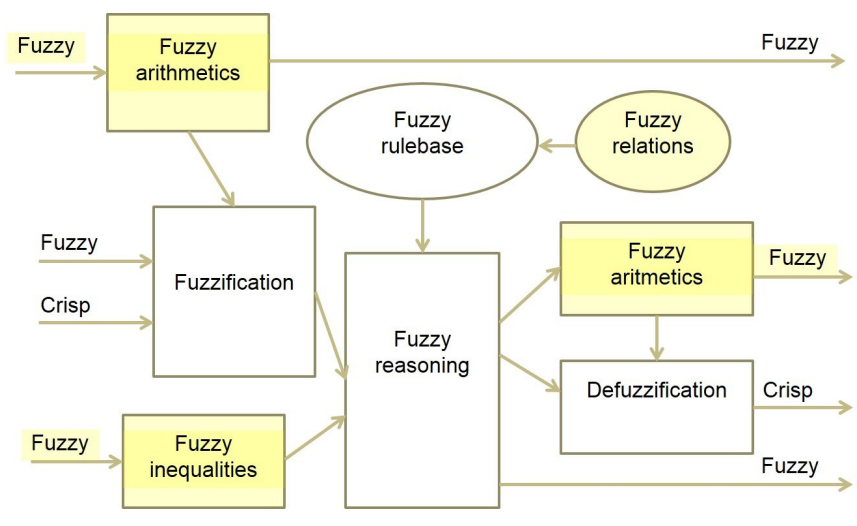

Fig. 4 Combined fuzzy set system

Source: [22].

\section{Fuzzy rule-based solutions}

In the combined systems, the fuzzy inputs can be fuzzy numbers or crisp inputs processed by fuzzy scaling functions (Fig. 4). The results can be defuzzified to crisp values, processed with fuzzy arithmetics or passed to other fuzzy set systems.

Type-2 fuzzy models introduced by Zadeh in 1975 take into account uncertainty about the membership function [49]. Most systems based on interval type-2 fuzzy sets are reduced to an interval-valued type-1 fuzzy set. Special cases of fuzzy linguistic equation models, which can be understood as linguistic Takagi-Sugeno (LTS) type fuzzy models, are robust solutions for applications where the same variables can be used for defining operating areas and in the submodels. No special smoothing algorithms are needed [50].

\section{OPERATION AND MAINTMENCE MANAGEMENT}

The nonlinear scaling approach is the basis of the consistent natural language interface.

\section{Monitoring and control}

The keys of the natural language interface are the monotonously increasing, nonlinear scaling functions, which are obtained by generalized norms and moments or defined manually based on domain expertise. The variable specific parameters can be recursively updated by using the corresponding norms and new data samples. Also the orders of the norms can be updated after drastic changes. Since the parameters specific to operating conditions, some changes can be taken into account switching the parameter sets. Uncertainty, fluctuations and confidence in 
results are estimated by a difference of norms of high positive and negative order, respectively. [39]

Feature levels, uncertainty, trends, trend episodes and severity can be evaluated by using scaled values, fluctuation, trend indices and derivatives of trend indices (Table 2).

Table 2

Monitoring interface

\begin{tabular}{lcccc}
\hline \multicolumn{1}{c}{ Task } & \multicolumn{3}{c}{ Features / indices } \\
\hline & $\begin{array}{c}\text { Scaled } \\
\text { value }\end{array}$ & $\begin{array}{c}\text { Variation/ } \\
\text { Fluctuation }\end{array}$ & $\begin{array}{c}\text { Trend } \\
\text { index }\end{array}$ & $\begin{array}{c}\text { Derivative } \\
\text { of trend index }\end{array}$ \\
Level & $\mathrm{x}$ & $\mathrm{x}$ & $\mathrm{x}$ & \\
Uncertainty & & $\mathrm{x}$ & $\mathrm{x}$ \\
$\begin{array}{l}\text { Trend } \\
\text { Trend } \\
\text { episodes }\end{array}$ & & & $\mathrm{x}$ & \\
$\begin{array}{l}\text { Trend } \\
\text { severity }\end{array}$ & $\mathrm{x}$ & & & $\mathrm{x}$ \\
\hline
\end{tabular}

Source: [39].

All indices are in the range $[-2,2]$ and interpreted in natural language labels, e.g. \{very low, low, normal, high, very high\}. The trend index $I_{j}^{T}(k)$ represents levels ffast decrease, decrease, constant, increase, fast increase\}, the derivative $\Delta I_{j}^{T}(k)$ levels ffast accelerating decrease, accelerating decrease, constant change, accelerating increase, fast accelerating increase $\}$ and the deviation $I_{j}^{D}(k)$ index \{serious decrease, decrease, normal, increase, serious increase\}, respectively. The fuzzy partition of all these can be refined by using more levels.

Advanced signal processing and feature extraction is combined with nonlinear scaling to obtain condition and stress indices in [33]. More information can be collected with reliability-centered maintenance (RCM) [5], and finally, all this can be monitored with statistical process control (SPC) [40].

Increased computational power in small programmable controllers and sensors open new possibilities for the efficient on-site calculations. Programmable automation controllers (PACs) make the algorithm testing efficient since the software can be updated easily and measurement setup can be customized. Several aspects connected to on-site calculations present a method for extracting meaningful numbers from high frequency vibration data [51,52].

The monitoring interface is aimed to utilize on-line measurements in stabilizing, optimizing and coordinating control.

\section{Control strategies and Maintenance}

Process control systems in industry include centralized or decentralized process controllers coupled with hosts, workstations and several process control and instrumentation devices, such as field devices. Applications are related to business functions in Enterprise resource planning (ERP) or maintenance functions in computerized maintenance management systems (CMMS). Smart field devices can in- clude equipment monitoring applications which are used to help monitor and maintain the devices [23].

Maintenance information is collected from various sources: condition monitoring measurements, performance indicators, including harmonized indicators, key performance indicators and overall equipment effectiveness (OEE). The systems include a huge amount of event information, which is not necessarily in a numeric form. The natural language information can be understood in the range $[-2,2]$ through linguistic levels and modifiers related [42].

At this level, the temporal analysis and uncertainty processing become important in detecting operating conditions. Model-based predictions and recursive updates of the parameters are needed in decision making, where the adaption of the control strategies is used in scheduling the condition-based maintenance actions.

\section{Management}

Performance indicators are specific for different industrial areas $[8,12]$. The nonlinear scaling brings the performance levels to a consistent range, which can be understood in linguistic terms. The levels and their improvements are represented in natural language, e.g. 'excellent improvement from poor performance to good performance' [5]. Aggregation is needed for the information obtained from other levels. Uncertainty processing is increasingly important in this level.

\section{CONCLUSIONS}

The nonlinear scaling approach is the main part of the data processing chain which is the integrating part of the natural language interface. The calculations are done in numeric forms, but the levels and all the indices based on them can be represented in natural language. The system includes integrated temporal analysis and uncertainty processing which facilitates the efficient use of domain expertise.

\section{ACKNOWLEDGMENT}

The author would like to thank the research program "Measurement, Monitoring and Environmental Efficiency Assesment (MMEA)" funded by the TEKES

(the Finnish Funding Agency for Technology and Innovation) and the Artemis Innovation Pilot project "Production and energy system automation and Intelligent-Built (Arrowhead)".

\section{REFERENCES}

[1] S. Lahdelma and E.K. Juuso, "Advanced signal processing and fault diagnosis in condition monitoring", Insight, vol. 49, no. 12, pp. 719-725, 2007.

[2] S. Lahdelma and E.K. Juuso, "Signal processing and feature extraction by using real order derivatives and generalised norms. Part 1: Methodology", The International Journal of Condition Monitoring, vol. 1, no. 2, pp. 46-53, 2011.

[3] S. Lahdelma and E.K. Juuso, "Signal processing and feature extraction by using real order derivatives and generalised norms. Part 2: Applications", The International Journal of Condition Monitoring, vol. 1, no. 2, pp. 54-66, 2011.

[4] E.K. Juuso and S. Lahdelma, "Intelligent scaling of features in fault diagnosis", in 7th Int. Conf. on Condition Monitoring and Machinery Failure Prevention Technol- 
ogies, Stratford-upon-Avon, United Kingdom, vol. 2, 2010, pp. 1358-1372.

[5] E.K. Juuso and S. Lahdelma, "Intelligent performance measures for condition-based maintenance", Journal of Quality in Maintenance Engineering, vol. 19, no.3, pp. 278-294, 2013.

[6] C. Olsson and T. Svantesson, "Harmonised maintenance and reliability indicators - compare apples to apples", Maintworld, vol. 1, no. 1, pp. 9-11, 2009.

[7] C. Idhammar, "The first world class maintenance organization", Maintworld, vol. 2, no. 2, pp. 52-53, 2010.

[8] A. Parida and U. Kumar, "Maintenance performance measurement - methods, tools and applications", Maintworld, vol. 1, no. 1, pp. 30-33, 2009.

[9] N.A. Al-Shammasi and S.S. Al-Shakhoyry, "Improving maintenance performance in Saudi Aramco", Maintworld, vol. 2, no. 2, pp. 6-9, 2010.

[10] SCEMM Keep It Running - Industrial Asset Management, Painoyhtymä, Loviisa, 1998.

[11] B. Hägg, "Maintenance - an investment in higher profitability", in Proc. of The Int. Conf. in Oulu, Oulu, Finland, 2010, pp. 7-14.

[12] P. Willmott, "Post the streamlining - 'where's your maintenance strategy now?", Maintworld, vol. 2, no. 1, pp. 16-22, 2010.

[13] S. Dash, R. Rengaswamy and V. Venkatasubramanian, "Fuzzy-logic based trend classification for fault diagnosis of chemical processes", Computers and Chemical Engineering, vol. 27, pp. 347-362, 2003.

[14] J.T.-Y. Cheung and G. Stephanopoulos, "Representation of process trends - part I. A formal representation framework", Computers and Chemical Engineering, vol. 14, no. 4-5, pp. 495-510, 1990.

[15] A.K.S. Jardine, D. Lin and D. Banjevic, "A review on machinery diagnostics and prognostics implementing condition-based maintenance", Mechanical Systems and Signal Processing, vol. 20, no. 7, pp. 1483-1510, 2006.

[16] A.H. Christer and W. Wang, "A model of condition monitoring inspection of production plant", International Journal of Production Research, vol. 30, no. 9, pp. 2199-2211, 1992.

[17] W. Wang, "A two-stage prognosis model in condition based maintenance", European Journal of Operational Research, vol. 182, no. 3, pp. 1177-1187, 2007.

[18] W. Schütz, "A history of fatigue, Engineering Fracture Mechanics", vol. 54, no. 2, pp. 263-300, 1996.

[19] A. Palmgren, "Die Lebensdauer von Kugellagern", Verfahrenstechnik, vol. 68, pp. 339-341, 1924.

[20] M.A. Miner, "Cumulative damage in fatigue", ASME Journal of Applied Mechanics, vol. 67, pp. 159-164, 1945.

[21] L.A. Zadeh, "Fuzzy sets", Information and Control, vol. 8, pp. 338-353, 1965.

[22] E.K. Juuso, "Intelligent Methods in Modelling and Simulation of Complex Systems", Simulation Notes Europe $S N E$, vol. 24, no. 1, pp. 1-10.

[23] E.K. Juuso and D. Galar, "Intelligent real-time risk analysis for machines and process devices", in Current Trends in Reliability, Availability, Maintainability and Safety: An Industry Perspective, K. Uday, A. Alireza, V.A. Kumar, V. Prabhakar, Eds. Cham: Springer International Publishing AG, pp. 229-240, 2016.

[24] K. Karioja and E.K. Juuso, "Generalised spectral norms - a new method for condition monitoring", Interna- tional Journal of Condition Monitoring, vol. 6, no. 1 , pp. 13-16, Mar. 2016.

[25] E.K. Juuso, "Integration of intelligent systems in development of smart adaptive systems", International Journal of Approximate Reasoning, vol. 35, no. 3, pp. 307-337, 2004.

[26] H.J. Zimmermann, Fuzzy set theory and its applications. Dordrecht: Kluwer Academic Publishers, 1992.

[27] E.K. Juuso, "Tuning of large-scale linguistic equation (LE) models with genetic algorithms", in Int. Conf. on Adaptive and Natural Computing Algorithms, Kuopio, Finland, 2009, pp. 161-170.

[28] T. Ahola, E.K. Juuso and K. Leiviskä, "Variable Selection and Grouping in a Paper Machine Application", International Journal of Computers, Communications \& Control, vol. 2, no. 2, pp. 111-120, 2007.

[29] VDI 2056 Beurteilungsmabstäbe für mechanische Schwingungen von Maschinen, VDI-Richtlinien, Oktober 1964.

[30] R.A. Collacott, Mechanical Fault Diagnosis and condition monitoring. London: Chapman and Hall, 1977.

[31] E.K. Juuso and S. Lahdelma, "Cavitation Indices in Power Control of Kaplan Water Turbines", in 6th Int. Conf. on Condition Monitoring and Machinery Failure Prevention Technologies, Dublin, Ireland, vol. 2, 2009, pp. 830-841.

[32] E.K. Juuso and M. Ruusunen, "Fatigue prediction with intelligent stress indices based on torque measurements in a rolling mill", in 10th Int. Conf. on Condition Monitoring and Machinery Failure Prevention Technologies, Krakow, Poland, vol. 1, 2013, pp. 460-471.

[33] E.K. Juuso, "Intelligent indices for online monitoring of stress and condition", in 11th Int. Conf. on Condition Monitoring and Machinery Failure Prevention Technologies, Manchester, United Kingdom, vol. 1, 2014, pp. 637-648.

[34] J. Laurila, A. Koistinen, E.K. Juuso and T. Liedes, "Monitoring of a rod mill using advanced feature extraction methods", in 12th Int. Conf. on Condition Monitoring and Machinery Failure Prevention Technologies, Oxford, United Kingdom, 2015, pp. 580-590.

[35] A. Koistinen, J. Laurila and E.K. Juuso, "Rod mill liner monitoring using cumulative stress", in 13th Int. Conf. on Condition Monitoring and Machinery Failure Prevention Technologies, Paris, France, 2016, pp. 131142.

[36] J. Nissilä, S. Lahdelma and J. Laurila, “Condition monitoring of the front axle of a load haul dumper with real order derivatives and generalised norms", in 11th Int. Conf. on Condition Monitoring and Machinery Failure Prevention Technologies, Manchester, United Kingdom, vol. 1, 2014, pp. 407-426.

[37] E.K. Juuso, "Model-based adaptation of intelligent controllers of solar collector fields", in 7th Vienna Symp. on Mathematical Modelling, Vienna, Austria, vol. 7, 2012, pp. 979-984.

[38] E.K. Juuso, "Intelligent Trend Indices in Detecting Changes of Operating Conditions", in 13th Int. Conf. on Computer Modelling and Simulation, Cambridge, United Kingdom, 2011, pp. 162-167.

[39] E.K. Juuso, "Informative process monitoring with a natural language interface", in 18th Int. Conf. on Modelling and Simulation, Rome, Italy 2016, pp. 105-110.

[40] E.K. Juuso, "Recursive Data Analysis and Modelling in Prognostics", in 12th Int. Conf. on Condition Monitor- 
ing and Machinery Failure Prevention Technologies, Oxford, UK, 2015, pp. 560-567.

[41] E.K. Juuso and M. Ruusunen, "Stress Indices in Fatigue Prediction", in Maintenance, Condition Monitoring and Diagnostics \& Maintenance Performance Measurement and Management, Oulu, Finland, 2015, pp. 89-96.

[42] E.K. Juuso, "Generalised statistical process control (GSPC) in stress monitoring", IFAC-Papers OnLine, vol. 28, no. 17, pp. 207-212, 2015.

[43] E.K. Juuso, "Integration of knowledge-based information in intelligent condition monitoring", in 9th Int. Conf. on Condition Monitoring and Machinery Failure Prevention Technologies, London, United Kingdom, vol. 1, 2012, pp. 217-228.

[44] M. De Cock and E.E. Kerre, "Fuzzy modifiers based on fuzzy relations", Information Sciences, vol. 160, no. 14, pp. 173-199, 2004.

[45] R.E. Moore, Interval Analysis. Englewood Cliffs (NJ): Prentice Hall, 1966.

[46] J.J. Buckley and T. Feuring, "Universal approximators for fuzzy functions", Fuzzy Sets and Systems, vol. 113, pp. 411-415, 2000.

[47] J.J. Buckley and Y. Hayashi, "Can neural nets be universal approximators for fuzzy functions?", Fuzzy Sets and Systems, vol. 101, pp. 323-330, 1999.

\section{D.Sc. Esko K. Juuso}

Control Engineering, Faculty of Technology

Pentti Kaiteran katu 1, 90014 Oulu, FINLAND

e-mail: esko.juuso@oulu.fi
[48] J.J. Buckley and Y. Qu, "On using $\alpha$-cuts to evaluate fuzzy equations", Fuzzy Sets and System, vol. 38, no. 3, pp. 309-312, 1990.

[49] J.M. Mendel, "Advances in type-2 fuzzy sets and systems", Information Sciences, vol. 177, no. 1, pp. 84 110, 2007.

[50] E.K. Juuso, "Development of Multiple Linguistic Equation Models with Takagi-Sugeno Type Fuzzy Models", in Int. Fuzzy Systems Association WORLD CONGR. \& European Society for Fuzzy Logic and Technology CONF., Lisbon, Portugal, 2009, pp. 1779-1784.

[51] A. Koistinen and E.K. Juuso, "On-site calculations of generalised norms for maintenance and operational monitoring", in Maintenance, Condition Monitoring and Diagnostics \& Maintenance Performance Measurement and Management, Oulu, Finland, 2015, pp. 107-112.

[52] A. Koistinen and E.K. Juuso, "Information from Centralized Database to Support Local Calculations in Condition Monitoring", in 9th EUROSIM Congr. on Modelling and Simulation, Oulu, Finland, 2016, pp. 10491054. 